\title{
Harmonically Trapped Bosons on the Sierpiński Carpet
}

\author{
A. ROVENCHAK \\ Department for Theoretical Physics, Ivan Franko National University of Lviv \\ 12 Drahomanov St., UA-79005, Lviv, Ukraine \\ (Received February 11, 2010; revised version May 26, 2010; in final form June 2, 2010)

\begin{abstract}
An ideal Bose-gas confined in a harmonic potential is studied. The thermodynamic properties of the said system are obtained in a general case of $D$ dimensions, where $D$ can be fractional (this corresponds to, e.g., porous medium). For the critical temperature, expansions with respect to the number of particles in the system are obtained. Numerical study is made for $D=\ln 8 / \ln 3$ corresponding to the dimensionality of the so-called Sierpiński carpet.
\end{abstract}

PACS numbers: 05.30.Jp, 67.85.-d, 64.60.an

\section{Introduction}

The achievement of the Bose-Einstein condensation (BEC) in ultracold trapped alkali gases $[1,2]$ stimulated the studies of the properties of trapped degenerate bosonic systems at various conditions. In particular, the effects of a finite number of particles on BEC of an ideal gas was discussed in theoretical works [3-7], where the corrections to the bulk properties were found. Bose-systems in porous medium are also under extensive study, in particular, $\mathrm{He}^{4}$ properties in porous glasses [8], aerogel $[9,10]$, nanoporous gelsil glass [11]. It is suggested that the presence of a porous medium can be analyzed by finding an analogy with fractional dimensionality [12-14]. This concept also appears effectively in the studies of systems confined in external potentials, cf. $[15,16]$. An approach to the problem of a finite bosonic system for a general case of $D$ dimensions with $D$ not necessarily being integer is given in this work.

The organization of the paper is as follows. In Sect. 2 the calculation scheme is given and formulae for numerical calculations are written. Semiclassical approach is also analyzed. The corrections to the transition temperature due to the finite number of particles are found analytically in Sect. 3. Thermodynamic properties of a bosonic system confined by a harmonic potential are calculated numerically in Sect. 4 for a particular case of a fractional $D$ corresponding to the Sierpiński carpet.

\section{General calculation scheme}

For the calculation of thermodynamic functions the following simple procedure is used. The number of particles $N$ is related to the fugacity $z$ and temperature $T$ as

$$
N=N_{0}+\sum_{n=1}^{\infty} \frac{g_{n}}{z^{-1} \mathrm{e}^{\varepsilon_{n} / T}-1},
$$

where $N_{0}=z /(1-z)$ is the occupation of the lowest energy level, $\varepsilon_{n}$ is the single-particle energy spectrum, and $g_{n}$ is the degeneracy of the $n$-th level. Let us note that $N_{0}$ is a macroscopic number only if the Bose-condensation occurs.

Equation (1) gives $z$ as an implicit function of $N$ and $T$. It can be used for the calculation of energy

$$
E=\sum_{n=1}^{\infty} \frac{\varepsilon_{n} g_{n}}{z^{-1} \mathrm{e}^{\varepsilon_{n} / T}-1},
$$

where the lowest energy level is taken zero. The temperature derivative of the energy is the heat capacity $C=\mathrm{d} E / \mathrm{d} T$.

\subsection{System of oscillators in $D$ dimensions}

Consider a system of $N$ harmonic oscillators confined in isotropic harmonic trap. The single-particle energy spectrum is given by $\varepsilon_{n}=\hbar \omega n$. In one dimension (1D), the degeneracy $g_{n}=1$, in $2 \mathrm{D} g_{n}=n+1$, and in $3 \mathrm{D}$ $g_{n}=(n+2)(n+1) / 2$. That is, the degeneracy in $D$ dimensions is given by the binomial coefficient

$$
g_{n}=C_{n+D-1}^{D-1}=\frac{(n+D-1) !}{n !(D-1) !} .
$$

A natural generalization of the above expression for a continuous $D$ is as follows, cf. [17]:

$$
g_{n}=\frac{\Gamma(n+D)}{\Gamma(n+1) \Gamma(D)} .
$$

That is, Eq. (1) becomes in this case

$$
N=\frac{z}{1-z}+\frac{z}{\Gamma(D)} \sum_{n=1}^{\infty} \frac{\Gamma(n+D)}{\Gamma(n+1)} \frac{1}{\mathrm{e}^{\hbar \omega n / T}-z} .
$$

One can also write a similar expression for the energy from Eq. (2).

\subsection{Semiclassical approach}

In the semiclassical approach, the discreteness of the energy levels is neglected, and the domain of the space accessible for a particle in an external field is defined by the classical turning points [15]. For the oscillator 
problem, the particle energy is given by $\varepsilon=p^{2} / 2 m+$ $m \omega^{2} q^{2} / 2$, where $p$ is the momentum absolute value and $q$ is that of the coordinate.

After simple transformations [15], one can obtain an analogue of Eq. (1), cf. also [13]:

$$
N=N_{0}+\left(\frac{T}{\hbar \omega}\right)^{D} \operatorname{Li}_{D} z
$$

where $\mathrm{Li}_{D} z$ is the polylogarithm function: $\mathrm{Li}_{D} z=$ $\sum_{n=1}^{\infty} \frac{z^{n}}{n^{D}}$, which becomes Riemann's zeta if $z=1$ : $\operatorname{Li}_{D} 1=\zeta(D)$.

Critical temperature $T_{\mathrm{c}}$ (the temperature at which the Bose-condensation starts) is defined by two conditions, $N_{0}=0$ and $z=1$. From Eq. (6) one thus obtains

$$
T_{\mathrm{c}}=\hbar \omega\left[\frac{N}{\zeta(D)}\right]^{1 / D} \text {. }
$$

Let us note that the above result is valid only for $D>1$ and there is no indication of the Bose-condensation in harmonic trap for $D \leq 1$ in the semiclassical approach.

The condensate fraction is given by

$$
\frac{N_{0}}{n}=1-\left(\frac{T}{T_{\mathrm{c}}}\right)^{D}
$$

and the energy equals

$$
E=\hbar \omega D\left(\frac{T}{\hbar \omega}\right)^{D+1} \operatorname{Li}_{D+1} z .
$$

At $T<T_{\mathrm{c}}$ the heat capacity is

$$
C=D(D+1)\left(\frac{T}{\hbar \omega}\right)^{D} \zeta(D+1) .
$$

At $T>T_{\mathrm{c}}$, Eq. (6) for $z$ should be solved numerically.

\section{3. $1 / N$-expansions for critical temperature}

Before proceeding to the exact calculations using Eq. (5) it is worth to note what is the thermodynamic limit for the problem under consideration. A hint might be seen in Eq. (7): $T_{\mathrm{c}}$ remains constant if $\omega N^{1 / D}=$ const. This is in fact the thermodynamic limit condition and such a relation might be obtained from different considerations [18]. To simplify the calculations it is convenient to use further $\hbar \omega$ as a unit for both energy and temperature. Also, the following notation will be used:

$$
T_{0}=\left(\frac{1}{\zeta(D)}\right)^{1 / D}
$$

The critical temperature of the system with discrete spectrum is thus defined from:

$$
\begin{aligned}
& N=\frac{1}{\Gamma(D)} \sum_{n=1}^{\infty} \frac{\Gamma(n+D)}{\Gamma(n+1)} \frac{1}{\mathrm{e}^{n / T_{\mathrm{c}}}-1}, \\
& T_{\mathrm{c}}=T_{0} N^{1 / D} .
\end{aligned}
$$

To handle this expression, one can use the Stirling formula for the gamma-functions and obtain an asymptotic expansion

$$
\frac{\Gamma(n+D)}{\Gamma(n+1)} \approx n^{D-1}\left[1+\frac{1}{n} \frac{D(D-1)}{2}+\ldots\right] .
$$

In the limit of large $N$ the summation of the first term can be done using the Euler-Maclaurin summation formula

$$
\begin{aligned}
& \sum_{n=1}^{\infty} \frac{n^{D-1}}{\mathrm{e}^{n / T_{\mathrm{c}}}-1}=T_{\mathrm{c}}^{D} \int_{1 / T_{\mathrm{c}}}^{\infty} \frac{x^{D-1} \mathrm{~d} x}{\mathrm{e}^{x}-1}+\frac{1}{2} T_{\mathrm{c}}+\ldots \\
& \quad=T_{\mathrm{c}}^{D} \int_{0}^{\infty} \frac{x^{D-1} \mathrm{~d} x}{\mathrm{e}^{x}-1}-T_{\mathrm{c}}^{D} \int_{0}^{1 / T_{\mathrm{c}}} \frac{x^{D-1} \mathrm{~d} x}{\mathrm{e}^{x}-1} \\
& \quad+\frac{1}{2} T_{\mathrm{c}}+\ldots \\
& \quad=T_{\mathrm{c}}^{D} \Gamma(D) \zeta(D)+T_{\mathrm{c}}\left[\frac{1}{2}+\frac{1}{D-1}+\ldots\right]+o\left(T_{\mathrm{c}}\right),
\end{aligned}
$$

where ... denotes small corrections from the 2 nd and next terms in the Euler-Maclaurin formula and $o\left(T_{c}\right)$ means the items which can be neglected comparing to $T_{c}$.

The second term in Eq. (13) gives the following contribution:

$$
\begin{aligned}
& \sum_{n=1}^{\infty} \frac{n^{D-2}}{\mathrm{e}^{n / T_{\mathrm{c}}}-1}=T_{\mathrm{c}} \sum_{n=1}^{\infty} n^{D-3}+o\left(T_{\mathrm{c}}\right) \\
& \quad=T_{\mathrm{c}} \zeta(3-D)+o\left(T_{\mathrm{c}}\right) .
\end{aligned}
$$

This expansion is correct for $D<2$. For other values the estimation can be done in a slightly different way.

The remaining terms in Eq. (13) can be evaluated as shown above, however, they give small corrections and can be neglected.

Collecting the calculated sums, one can write the expansion for the critical temperature $T_{\mathrm{c}}^{(N)}=T_{0}^{(N)} N^{1 / D}$ of the $N$-particle system

$$
T_{0}^{(N)}=\left(\frac{1}{\zeta(D)}\right)^{1 / D}\left[1-B_{D} N^{1 / D-1}+o\left(N^{1 / D-1}\right)\right],
$$

where

$$
\begin{aligned}
& B_{D}=\left(\frac{1}{\zeta(D)}\right)^{1 / D} \frac{1}{2 \Gamma(D+1)} \\
& \quad \times\left[1-\frac{2}{D-1}+D(D-1) \zeta(3-D)+\ldots\right] .
\end{aligned}
$$

\section{Numerical results and discussion}

The numerical analysis is made for the dimensionality corresponding to the Sierpinski carpet, a fractal object with the Hausdorff dimension $D=\ln 8 / \ln 3=$ $1.892789 \ldots$. As fractals of this type lack translational symmetry, the results of the calculations are not to be applied directly for such objects - this value of $D$ was chosen arbitrarily. The results of the direct solution of Eqs. (1), (2) are presented in Figs. 1, 2 for different $N$ in comparison with semiclassical results. For better comprehension, the temperature is scaled for different $N$ using the factor $N^{1 / D}$ (see Sect. 3 for details).

The obtained results are consistent with those of other authors, in particular [3], where the calculations were made for one and three dimensions, and [19] with the analysis of one and two dimensions, as well as with Monte 


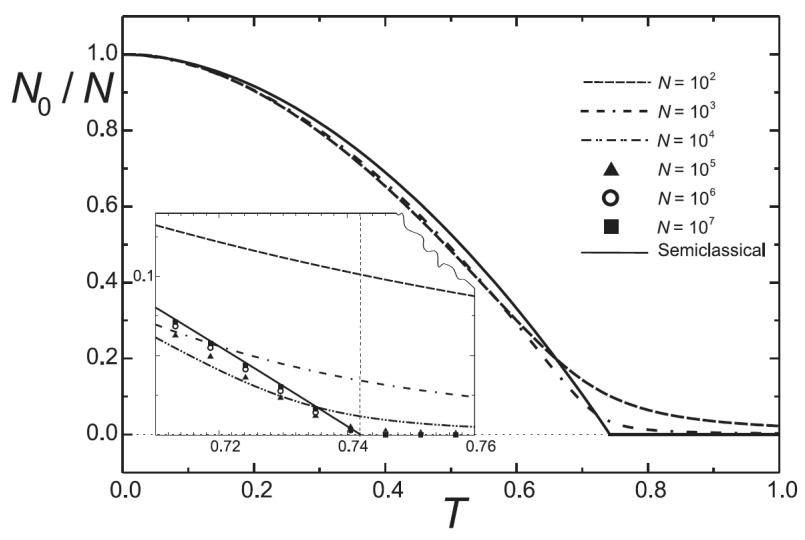

Fig. 1. Condensate fraction of the ideal harmonically trapped Bose gas on the Sierpiński carpet. Inset shows the vicinity of the critical temperature (enlarged view).

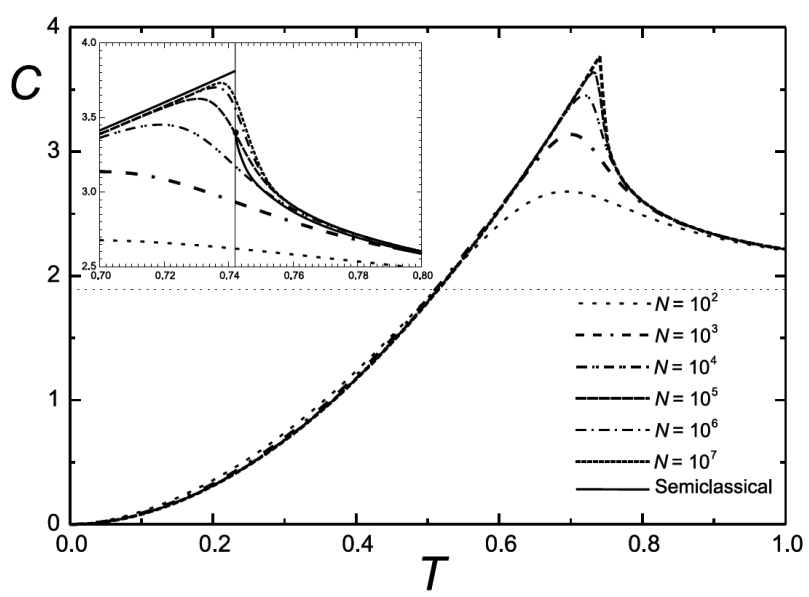

Fig. 2. Specific heat of the ideal harmonically trapped Bose gas on the Sierpiński carpet. The horizontal dotted line corresponds to the asymptotic value $C=D=$ $1.89 \ldots$. Inset shows the vicinity of the critical temperature (enlarged view). Let us note the gap in the semiclassical specific heat at the critical temperature.

Carlo simulations of a two-dimensional Bose gas [20]. Typical behavior of the heat capacity and condensate fraction in the vicinity of the transition temperature consists in smearing of the transition region and smoothing the respective curves as the number of particles decreases, as well as in the lowering of the transition temperature, cf. e.g. [4]. The results also correlate with the analysis of trapped weakly interacting bosons in two dimensions [21] and with the Hartree-Fock-Bogoliubov treatment of a quasi-two-dimensional trapped Bose system [22]. One should note that a harmonically trapped system in $D$ dimensions effectively corresponds (in the semiclassical approach) to an ideal system in a $2 D$-dimensional rigid box, cf. [16].

\section{Summary}

To summarize, the deviation from the semiclassical approach is analyzed and the corrections due to the discreteness of the energy levels are found. Obtained analytical dependence for the critical temperature are useful for the study of the influence of finite number of particles on physical properties of bosonic systems. Numerical results show that discreteness of the energy levels must be taken into consideration when analyzing in detail the temperature domain in the vicinity of the Bose-condensation point.

\section{References}

[1] M.H. Anderson, J.R. Ensher, M.R. Matthews, C.E. Wieman, E.A. Cornell, Science 269, 198 (1995).

[2] K.B. Davis, M.-O. Mewes, M.R. Andrews, N.J. van Druten, D.S. Durfee, D.M. Kurn, W. Ketterle, Phys. Rev. Lett. 75, 3969 (1995).

[3] W. Ketterle, N.J. van Druten, Phys. Rev. A 54, 656 (1996).

[4] W. Deng, P.M. Hui, Solid State Commun. 104, 729 (1997).

[5] R.K. Pathria, Phys. Rev. A 58, 1490 (1998).

[6] M. Li, L. Chen, J. Chen, Z. Yan, C. Chen, Phys. Rev. A 60, 4168 (1999).

[7] I.O. Vakarchuk, A.A. Rovenchak, Condens. Matter Phys. 4, 431 (2001).

[8] D. Finotello, K.A. Gillis, A. Wong, M.H.W. Chan, Phys. Rev. Lett. 61, 1954 (1988).

[9] M. Chan, N. Mulders, J. Reppy, Physics Today 49, 30 (1996).

[10] R.E. Boltnev, I.B. Bykhalo, S.V. Ivashin, I.N. Krushinskaya, L.P. Mezhov-Deglin, J. Low Temp. Phys. 150, 511 (2008).

[11] K. Yamamoto, Y. Shibayama, K. Shirahama, J. Low Temp. Phys. 150, 353 (2008); Phys. Rev. Lett. 100, 195301 (2008).

[12] E. Courtens, R. Vacher, Proc. R. Soc. Lond. Ser. A 423, 55 (1989).

[13] S.-H. Kim, Ch.K. Kim, K. Nahm, J. Phys., Condens. Matter 11, 10269 (1999).

[14] J. Kou, F. Wu, H. Lu, Y. Xu, F. Song, Phys. Lett. A 374, 62 (2009).

[15] V. Bagnato, D. Kleppner, Phys. Rev. A 44, 7439 (1991).

[16] L. Salasnich, J. Math. Phys. 41, 8016 (2000).

[17] V.P. Maslov, Theor. Math. Phys. 153, 1575 (2007).

[18] A. Posazhennikova, Rev. Mod. Phys. 78, 1111 (2006).

[19] G.-L. Ingold, A. Lambrecht, Eur. Phys. J. D 1, 2932 (1998).

[20] S. Pearson, T. Pang, Ch. Chen, Phys. Rev. A 58, 4811 (1998).

[21] M. Bayindir, B. Tanatar, Phys. Rev. A 58, 3134 (1998).

[22] Ch. Gies, B.P. van Zyl, S.A. Morgan, D.A.W. Hutchinson, Phys. Rev. A 69, 023616 (2004). 